\title{
Absence of mutations in the regulatory domain of the gap junction protein connexin 43 in patients with visceroatrial heterotaxy
}

\author{
Miranda Penman Splitt, Meng Y Tsai, John Burn, Judith A Goodship
}

\begin{abstract}
Objective-To determine the frequency of mutations in the regulatory domain of the gap junction protein connexin 43 in patients with visceroatrial heterotaxy. Design-Mutation screening of the terminal 200 base pairs of connexin43 gene coding sequence in a series of patients from tertiary care centres.

Patients-48 patients with visceroatrial heterotaxy attending UK Regional Paediatric Cardiology Centres.

Results-No changes from the published connexin 43 consensus sequence were found in any of the 48 patients studied. Conclusions-Germline mutations of the phosphorylation sites in the regulatory domain of the connexin 43 gene are rare in patients with visceroatrial heterotaxy.
\end{abstract}

(Heart 1997;77:369-370)

Keywords: visceroatrial heterotaxy; isomerism sequence; connexin 43; asplenia; polysplenia

Connexin 43 is the major gap junction protein in the adult heart. ${ }^{1}$ Evidence for spatial and temporal restriction of expression during vertebrate development suggests it plays an important role in various aspects of development. ${ }^{2}$ The hypothesis that mutations in the connexin 43 gene which altered sites critical to the regulation of connexin 43 expression would lead to functional or developmental abnormalities led Britz-Cunningham et al to search for mutations in patients with congenital heart malformations. ${ }^{3}$ They studied 30 patients with a variety of congenital heart defects and found mutations in seven. Six of these patients had complex heart defects and visceroatrial heterotaxy (VAH), while the seventh had an atrial septal defect. No mutations were found in the other 23 patients without defects of laterality, nor in 25 normal subjects. All six children with

Department of Human Genetics, University of Newcastle upon Tyne, Newcastle upon Tyne, United Kingdom M Penman Splitt M Y Tsai

J Burn

J A Goodship

Correspondence to:

Dr M Penman Splitt, Department of Human Genetics, 19/20 Claremont Place, Newcastle upon Tyne, NE2 4AA, United Kingdom. Accepted for publication 6 November 1996
VAH had single base substitutions of one or more phosphorylatable serine or threonine residues. Four had two independent mutations suggesting a recessive disorder. All mutations were in the last 200 base pairs of the coding sequence. Britz-Cunningham's findings suggest that mutations in the regulatory domain of connexin 43 will be a common finding in patients with VAH.

In this study we set out to document the frequency of these mutations in a large series of children with complex heart malformations and evidence of laterality disturbance.
Methods

SUBJECTS

Forty eight patients with VAH and complex heart defects were studied. Patients were identified through regional paediatric cardiology centres and clinical information was obtained from the referring cardiologist, based on the combination of echocardiography, angiography, and findings at surgery.

Of the 48 patients three had an affected sibling. The rest were isolated cases. In the familial cases only the proband was tested. Fourteen patients were Pakistani, one was Gujarati Indian, and the remaining 33 were white European. The most frequent heart lesions seen were complete atrioventricular septal defect $(63 \%)$, common atrium (38\%), and pulmonary atresia or stenosis (58\%). Forty one patients had isomerism of the atrial appendages or bronchi or both: 14 had right isomerism and of these 10 were asplenic; 27 had left isomerism, with nine known to have polysplenia. Seven patients had VAH without atrial isomerism. Of these, two had siblings with isomerism sequence.

\section{DNA EXTRACTION}

Genomic DNA was extracted from whole blood or EBV transformed lymphoblastoid cell lines by the nucleon method (Scotlab).

PCR AMPLIFICATION OF CARBOXY-TERMINAL DOMAIN OF CONNEXIN43

Samples were subjected to two rounds of polymerase chain reaction (PCR; conditions available on request). The position and sequence of the primers used is shown in the figure. An intronic primer (primer 1) was necessary to avoid simultaneous amplification of a processed pseudogene. ${ }^{1}$ The first round used primers 1 and 2. Nested PCR amplification was performed with primers 3 and 2, using $2 \mu \mathrm{l}$ of the first round PCR product. Primer 2 was $5^{\prime}$ biotinylated to facilitate sequencing.

\section{SEQUENCING}

Sequencing was carried out using the chain termination method (Sequenase version 2.0 kit, United States Biochemicals) with primer 4. Reactions were carried out using biotinylated DNA attached to streptavidin beads (Dynal) in order to facilitate separation of the DNA strands after the sequencing reaction (details available on request). The labelling reaction using ${ }^{35} \mathrm{~S}$ ATP and termination reactions were carried out according to the manufacturer's instructions. The reactions were run on $6 \%$ acrylamide gels at $50-55^{\circ} \mathrm{C}$. In patients 


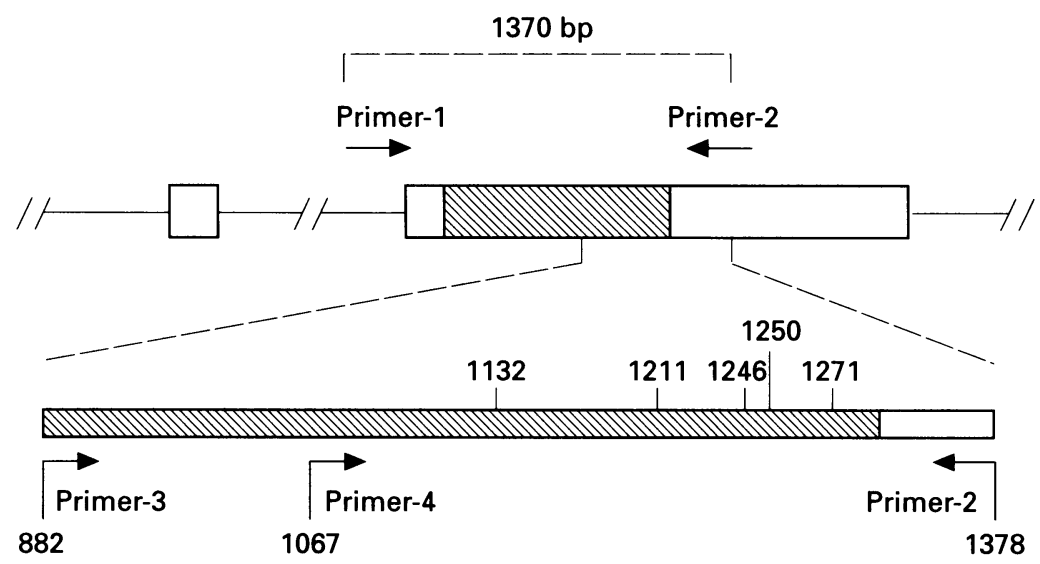

Connexin 43 genomic structure showing exons (boxes), coding region (hatched box), and introns (line). Numbers refer to nucleotide position in connexin 43 cDNA (GenBank accession No X52947) and show the positions of the five previously reported mutations and the start positions of PCR primers used (arrows). Primer sequences used were: primer 1, 5'GAAATACGTGAAACCGTTGG 3'; primer 2,

5'CCTGGTGCATTTCTACAGC 3'; primer 3, 5'CCTGGTGCATTTCTACAGC 3'; primer 4, 5'AAGCAAGTGAGCAAAACTGGCC 3'. Intronic sequence for the design of primer 1 was made available to us by Dr Glen Fishman, Albert Einstein College, New York.

where there was any doubt about the sequence the opposite DNA strand was also sequenced. The second round PCR reaction was repeated using $5^{\prime}$ biotinylated primer 3 , and primer 2 was used for sequencing.

\section{Results}

No changes from the predicted consensus sequence for connexin 43 gene (Genbank Accession No X52947) from codon 325 to the termination codon (codon 383) were found in any of the 48 patients studied. All the mutations detected by Britz-Cunningham et al lie within this $174 \mathrm{bp}$ region.

\section{Discussion}

The connexin 43 gene is a good candidate for congenital heart defects in humans. Two transgenic mouse models involving connexin 43 mutations have abnormal cardiac development. The first is a "knockout" in which the whole gene is disrupted and the animal does not express connexin 43 at all. ${ }^{4}$ Homozygotic mutant mice die at birth because of complete occlusion of the pulmonary outflow tract. They do not appear to have any other structural anomalies. The second mouse overproduces connexin 43 and is said to have defects in laterality including aberrant heart looping. ${ }^{5}$ This sounds a potentially good model for VAH in man. However, left right axis specification in vertebrates is a complex process involving a cascade of different developmental genes. ${ }^{6}$ Theoretically, a defect in any one of the many different genes in the pathway would be capable of producing laterality disturbance. Familial cases showing autosomal recessive, $\mathrm{X}$ linked, and autosomal dominant inheritance have all been recognised. ${ }^{7}$ In this context, Britz-Cunningham and colleagues' finding of mutations in connexin 43 in all six of their patients with VAH is surprising. ${ }^{8}$ They suggest that their patients may represent a distinct subtype in that they were selected through their need for heart transplantation and all had pulmonary atresia or stenosis. ${ }^{8}$ This seems an unlikely explanation. Pulmonary atresia and pulmonary stenosis are common defects in patients with VAH and were present in 12 of our 14 patients with right isomerism sequence, and in 12 of the 27 with left isomerism. Several of our patients have uncorrectable lesions and are awaiting heart transplant. Thus the two groups of patients do not appear to be phenotypically distinct. Given the evidence for clinical variability in familial cases of heterotaxy, the correctability of the heart lesion would not be expected to define a genetically distinct group. Our failure to detect mutations in connexin 43 in British patients is in keeping with Gebbia et al, who were unable to find mutations in 38 American patients. ${ }^{9}$

The major difference between our study and that of Britz-Cunningham et al was the tissue from which DNA was extracted. We studied DNA from white blood cells, while Britz-Cunningham used heart muscle. This raises the intriguing possibility of somatic mutation. This would have to have occurred before heart looping, that is, between conception and the fourth gestational week. Against this hypothesis is the finding of two independent mutations in opposite alleles in four of the six subjects they studied, and the fact that one case had an affected sibling. This possibility could easily be clarified by investigating the parents. ${ }^{8}$

We conclude that germline mutations of the regulatory phosphorylation sites in the connexin 43 gene are rare in patients with laterality disturbance. Until further evidence is available we would not consider it appropriate to institute mutation analysis of connexin43 as part of the routine management and genetic advice for families of children with laterality sequence.

Dr M Penman Splitt is funded by the British Heart Foundation and our group receives additional support from the Borwick Trust. We would like to thank the patients and their parent and all the Paediatric Cardiologists who supplied us with clinical information We are grateful to Dr Brett Casey, Baylo College of Medicine, Houston, for giving us the connexin 43 primers.

1 Fishman GI, Eddy RL, Shows TB, Rosenthal L, Leinwand LA. The human connexin gene family of gap junction proteins: distinct chromosomal locations but similar structures. Genomics 1991;10:250-6.

2 Ruangvoravat CP, Lo CW. Connexin 43 expression in the mouse embryo: localization of transcripts within developmentally significant domains. Dev Dyn 1992;194:261-81

3 Britz-Cunningham SH, Shah MM, Zuppan CW, Fletche WH. Mutations of the Connexin43 Gap-Junction in patients with heart malformations and defects of laterality. N Engl F Med 1995;332:1323-9.

4 Reaume AG, de Sousa PA, Kulkarni S, Langille BL, Zhu $\mathrm{D}$, Davies TC, et al. Cardiac malformation in neonatal mice lacking connexin43. Science 1995;267:1831-4.

5 Lo CW, Ewart JL, Sullivan R. Transgenic mouse models indicate a role for gap junctions in cell signalling events in early post implantation development [abstract]. Dev Biol 1995;170:740.

6 Yost HJ. Vertebrate left-right development. Cell 1995;82: 689-92.

7 Burn J. Disturbance of morphological laterality in humans. In: Bock GR, Marsh J, eds. Biological asymmetry and In: Bock GR, Marsh J, eds. Biological asym

8 Casey B, Ballabio A. Connexin 43 mutations in sporadic and Casey B, Ballabio A. Connexin 43 mutations in sporadic an familial defects of laterality. $N$ Engl F Med 1995;333:941

9 Gebbia M, Towbin JA, Casey B. Failure to detect connexin43 mutations in 38 cases of sporadic and familial
heterotaxy. Circulation 1996;94:1909-12. 